\title{
Limitations of Nanoparticles Size Characterization by Asymmetric Flow Field-Fractionation Coupled with Online Dynamic Light Scattering
}

\author{
Francesco Giorgi ${ }^{1}$ (D) Judith M. Curran ${ }^{1} \cdot$ Douglas Gilliland $^{2} \cdot$ Rita La Spina $^{2} \cdot$ Maurice Whelan $^{2} \cdot$ Eann A. Patterson ${ }^{1}$
}

Received: 15 September 2020 / Revised: 4 December 2020 / Accepted: 6 December 2020 / Published online: 5 January 2021

(c) The Author(s) 2020

\begin{abstract}
The development of reliable protocols suitable for the characterisation of the physical properties of nanoparticles in suspension is becoming crucial to assess the potential biological as well as toxicological impact of nanoparticles. Amongst sizing techniques, asymmetric flow field flow fractionation (AF4) coupled to online size detectors represents one of the most robust and flexible options to quantify the particle size distribution in suspension. However, size measurement uncertainties have been reported for on-line dynamic light scattering (DLS) detectors when coupled to AF4 systems. In this work we investigated the influence of the initial concentration of nanoparticles in suspension on the sizing capability of the asymmetric flow fieldflow fractionation technique coupled with an on-line dynamic light scattering detector and a UV-Visible spectrophotometer (UV) detector. Experiments were performed with suspensions of gold nanoparticles with a nominal diameter of $40 \mathrm{~nm}$ and $60 \mathrm{~nm}$ at a range of particle concentrations. The results obtained demonstrate that at low concentration of nanoparticles, the AF4-DLS combined technique fails to evaluate the real size of nanoparticles in suspension, detecting an apparent and progressive size increase as a function of the elution time and of the concentration of nanoparticles in suspension.
\end{abstract}

Keywords Asymmetric flow field-flow fractionation $\cdot$ Dynamic light scattering $\cdot$ Nanoparticles $\cdot$ Size characterization

\section{Introduction}

Asymmetric flow field flow fractionation (AF4) is a liquidbased fractionation technique which is commonly used in the separation and characterization of particle, polymer and protein suspensions. AF4 separates particles in suspension by the application of a cross-flow perpendicular to the direction of a longitudinal suspension flow through a thin ribbon-like separation channel. The high-dimensional aspect ratio of the separation channel causes the longitudinal suspension flow to develop a parabolic profile, with a

Supplementary Information The online version contains supplementary material available at https://doi.org/10.1007/s1033 7-020-03997-7.

Francesco Giorgi

Francesco.giorgi@liverpool.ac.uk

1 Faculty of Science and Engineering, University of Liverpool, Liverpool L69 3GH, UK

2 European Commission, Joint Research Centre (JRC), 21027 Ispra, Italy flow velocity that increases from a minimum value at the channel walls to a maximum value at the centre of the channel. The perpendicularly applied cross-flow forces a particle in suspension towards the bottom wall of the channel. A counteracting diffusive force arises due to the concentration gradient formed and drives the particles back towards the centre of the channel [1]. Once the equilibrium between the cross-flow force and the diffusion force is achieved, particles in suspension reside at a certain distance from the accumulation wall depending on their diffusion coefficient and size (Fig. 1). Smaller particles equilibrate higher up in the channel and experience a faster longitudinal flow, causing them to elute from the channel before the larger ones, thus allowing separation [2]. The size-separated suspension of particles can be then characterised and analysed by coupling AF4 to online detectors such as visible spectrophotometry (UV) to measure particle concentration and dynamic light scattering to provide particle size information. In this way it becomes possible to determine of the size distribution of the particles in suspension in relation to their mass/concentration [3]. It has been claimed that AF4 is able to separate particles with high resolution: within a broad size range 
Fig. 1 Schematic of the separation principle of asymmetric flow field flow fractionation [21]
C H A N N E L T O P

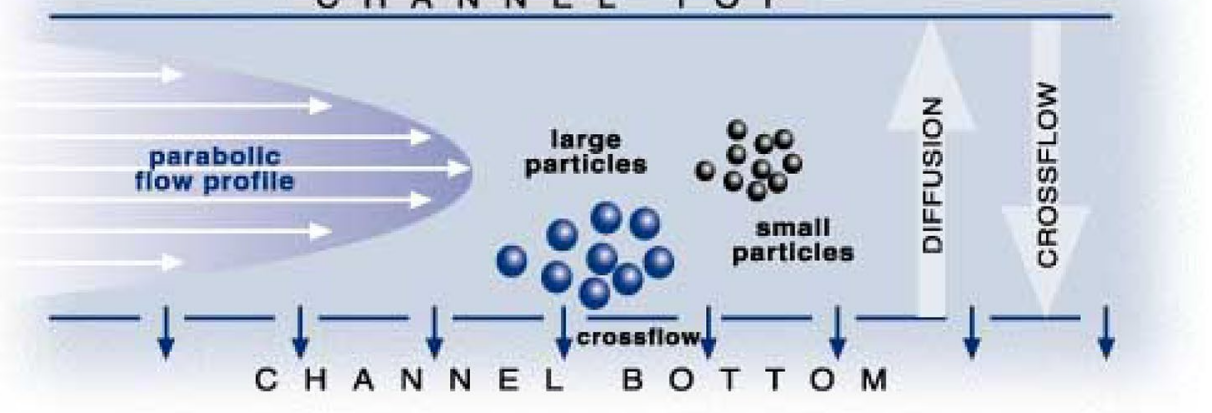

( $1 \mathrm{~nm}$ to $100 \mu \mathrm{m}$ in diameter) [4]; of a variety of materials (including polymers, organic and metallic particles, bacteria, viruses, proteins etc.) [4,5]; dispersed in aqueous and organic solvent carriers [1]. A typical fractogram generated by an AF4 coupled with online DLS and UV-Vis detectors for a single elution peak is presented in Fig. 2. The fractogram contains quantitative information about the suspension eluted by the $\mathrm{AF} 4$, providing the hydrodynamic diameter of the particles recorded by DLS and the absorbance recorded by UV-Vis spectrophotometer (directly linked to the concentration of particles in suspension [6]) as a function of the elution time. The Gaussian distribution of the absorbance as function of the elution time provides evidence that the concentration of particles in solution is lower at the beginning and at the end of the elution process. It can be noted that for low concentrations of particles in suspension (zones 1 and 3 ) the DLS detected an apparent and progressive increase in the size of the particles in suspension, returning a $U$-shaped distribution for the diameters as function of the elution time and of the concentration of particles in suspension. This phenomenon can be found in a significant number of studies in the literature describing the application of asymmetric flow field flow fractionation to separate and characterise particles of various sizes and materials [3,4,7-10]; however, a clear understanding of the factors regulating its appearance is still missing. Some studies in the literature suggest that the apparent progressive size increase detected by the DLS at low concentrations of particles (at the beginning and at the end of the absorbance distribution curve) can be related to the presence of larger aggregates in suspension. This
Fig. 2 Schematic fractogram generated by an AF4-DLS-UV system for a single elution peak. The fractogram can be divided in three zones according to the absorbance exhibited by the suspension of particles during the elution process. Zones 1 and 3 are associated with low particle concentration, while zone 2 is associated with high particle concentrations

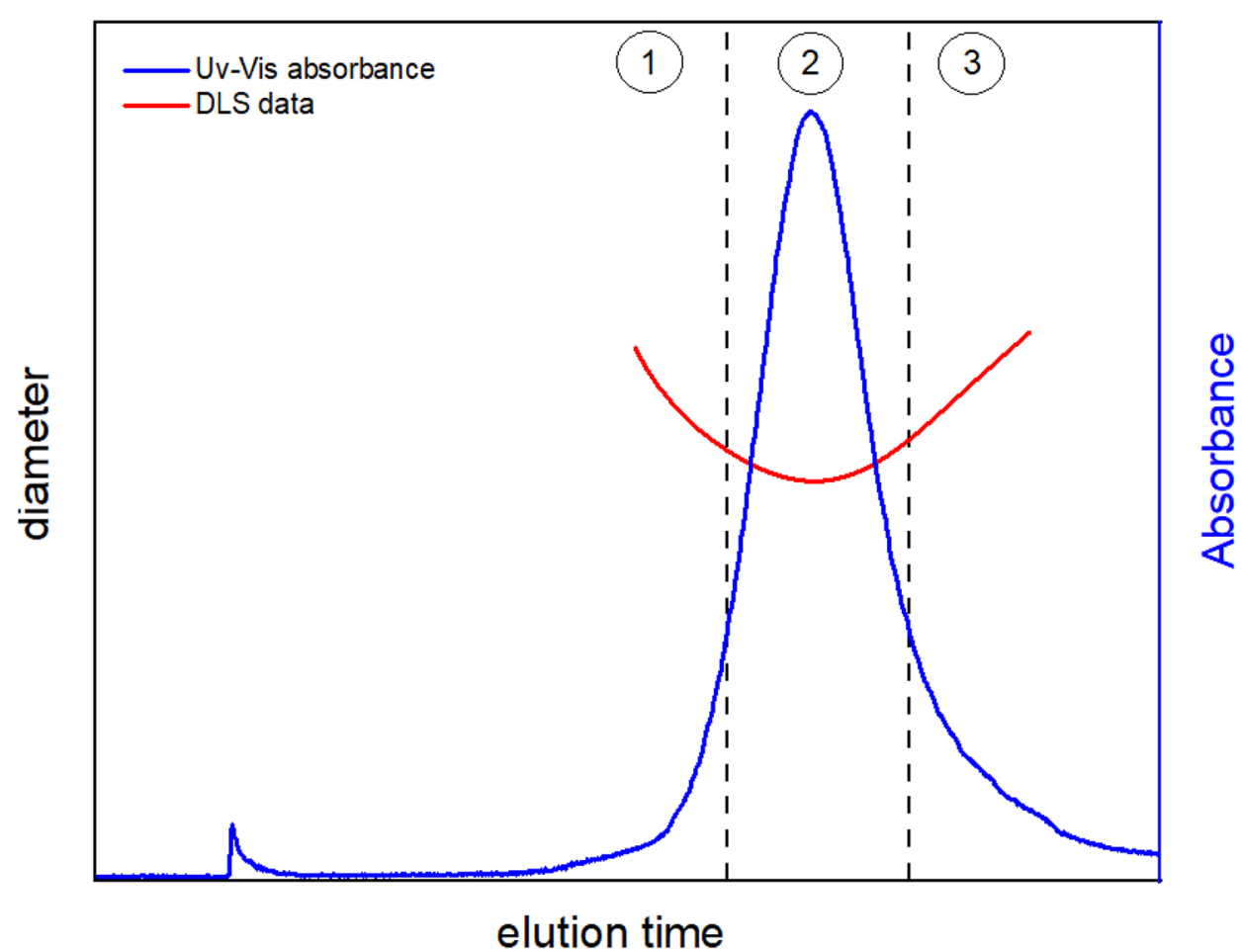


hypothesis could explain the trend towards an increase in diameter at the right tail of the concentration distribution but contradicts the operating principle of the AF4 for the left tail, because smaller particles are eluted before larger ones $[10,11]$. Other authors suggested that deviations between the real physical properties of the particles and the ones detected by the AF4-DLS-UV system are linked with a change in the surface shape of the particle during the separation and elution processes, but no experimental investigations support this hypothesis [8]. In this study, we investigated the influence of the initial concentration of nanoparticles in suspension on the sizing ability of an AF4-DLS-UV system, by testing suspensions of gold nanoparticles with a known nominal diameter of $40 \mathrm{~nm}$ and $60 \mathrm{~nm}$. The results obtained demonstrate that the sizing inaccuracy of the AF4-DLS-UV system is more evident for suspensions at the lowest initial concentration of nanoparticles of $10^{9}$ particles $\mathrm{mL}^{-1}$, and that the sizing accuracy of the AF4-DLS-UV system is directly linked with the initial concentration of nanoparticles in suspension. The size distribution of the same suspensions of nanoparticles was also evaluated by Centrifugal Liquid Sedimentation (CLS) analysis, to investigate whether the concentration of nanoparticles influences the sizing ability of a technique based on a different operating principle and theory. The CLS results show negligible influence of the initial working concentration of the particles on the sizing ability of the instrument. Hence, the CLS analysis represents a more robust method to quantify the size distribution of a suspension of particles within the concentration range tested.

\section{Materials and Methods}

Spherical citrate-capped gold nanoparticles $([\mathrm{Au}]=0.25 \mathrm{mM})$ were purchased from Sigma Aldrich (St. Louis, MO, USA), with nominal diameters of $40 \mathrm{~nm}$ and $60 \mathrm{~nm}$. The as-supplied concentrations were reduced by adding the concentrate to $2.5 \mathrm{mM}$ citrate solution as appropriate to obtain initial working concentrations of $10^{9}$ particles $\mathrm{mL}^{-1}, 10^{10}$ particles $\mathrm{mL}^{-1}$ and $10^{11}$ particles $\mathrm{mL}^{-1}$. Asymmetric flow field flow fractionation analysis was performed in an AF2000 Multiflow FFF system (Postnova Analytics) equipped with an on-line PN3212 spectrophotometer UV-Vis detector (Postnova Analytics) and with a DLS detector (Malvern). The AF4 channel had a $280 \mathrm{~mm}$ long separation channel, with a $350 \mu \mathrm{m}$ spacer.

A $10 \mathrm{kDa}$ cut-off membrane of regenerated cellulose and a $100 \mu \mathrm{l}$ injection loop were used. Phosphate buffer ( $1 \mathrm{mM}, \mathrm{pH}$ 7.4) was used as a carrier. The parameters of the AF4 separation were as follows: During a 5 min Injection and Focussing step the sample flow was $0.2 \mathrm{~mL} \mathrm{~min}^{-1}$ with a Focus Flow of $1.8 \mathrm{~mL} \mathrm{~min}{ }^{-1}$ and Cross flow of $1.5 \mathrm{~mL} \mathrm{~min}^{-1}$. This step was followed by $1 \mathrm{~min}$ transition step to the starting conditions of the elution. The elution step started with a Cross-flow of $1.5 \mathrm{~mL}$ which decreased as a power function (Exponent $=0.45$ ) to $0.1 \mathrm{~mL} \mathrm{~min}^{-1}$ over 35 min after which the cross flow was maintained constant for a further $10 \mathrm{~min}$. In each step the outflow to the detector flow was $0.5 \mathrm{~mL} \mathrm{~min}^{-1}$. The UV-Vis spectrophotometer wavelength was set to $525 \mathrm{~nm}$, corresponding to the maximum of the surface plasmon resonance band for $40 \mathrm{~nm}$ and $60 \mathrm{~nm}$ gold nanoparticles [12]. The suspensions of nanoparticles were injected into the separation chamber in ascending order of initial working concentration. Between each injection, the phosphate buffer was allowed to flow through the AF4-DLS-UV system for $10 \mathrm{~min}$, to remove any residual from the previous suspension. The parameters of the AF4 procedure used, including focusing time ( $5 \mathrm{~min}$ ) had previously been optimised systematically and evaluated for use in separating a range of $\mathrm{Au}, \mathrm{Ag}$ and $\mathrm{SiO} 2$ nanoparticles with sizes in the range of $10-110 \mathrm{~nm}[13,14]$.

The reliability of the data provided by the AF4-DLS-UV system was analysed by dividing the fractogram into three regions and evaluating the coefficient of variation of the DLS measurements for each suspension tested. The three regions of the fractogram were identified according to the absorbance detected by the UV-Vis spectrophotometer over the elution time in the following manner:

- Zones 1 and 3: regions of the fractogram, where the absorbance of the suspension is lower than the $30 \%$ of the maximum; and,

- Zone 2: region of the fractogram, where the absorbance of the suspension is higher than the $30 \%$ of the maximum.

The average and relative standard deviations of the diameters detected by the DLS for each suspension of nanoparticles tested are available in the supplementary material (Tables S1 and S2).

Centrifugal Liquid Sedimentation analysis was performed in a disk centrifuge DC24000UHR (CPS Instruments) using an 8-24 wt\% sucrose density gradients with a disc speed of $22,000 \mathrm{rpm}$. Each sample injection of $100 \mu \mathrm{L}$ was preceded by a calibration step performed using certified PVC particles with a weighted mean size of $237 \mathrm{~nm}$.

\section{Results and Discussion}

The DLS size measurements are shown in Fig. 3 as a function of the elution time, along with the relative coefficients of variation at the various stages of the elution process for each suspension of $40 \mathrm{~nm}$ gold nanoparticles tested. The complete fractogram for each suspension of particles tested is included in the supplementary material 
Fig. 3 Detected diameters of $40 \mathrm{~nm}$ gold nanoparticles (data points and left axis) in suspension at different concentrations as a function of the elution time and coefficient of variation (columns and right axis) for the three absorbance zones identified based on results from AF4-DLS-UV system

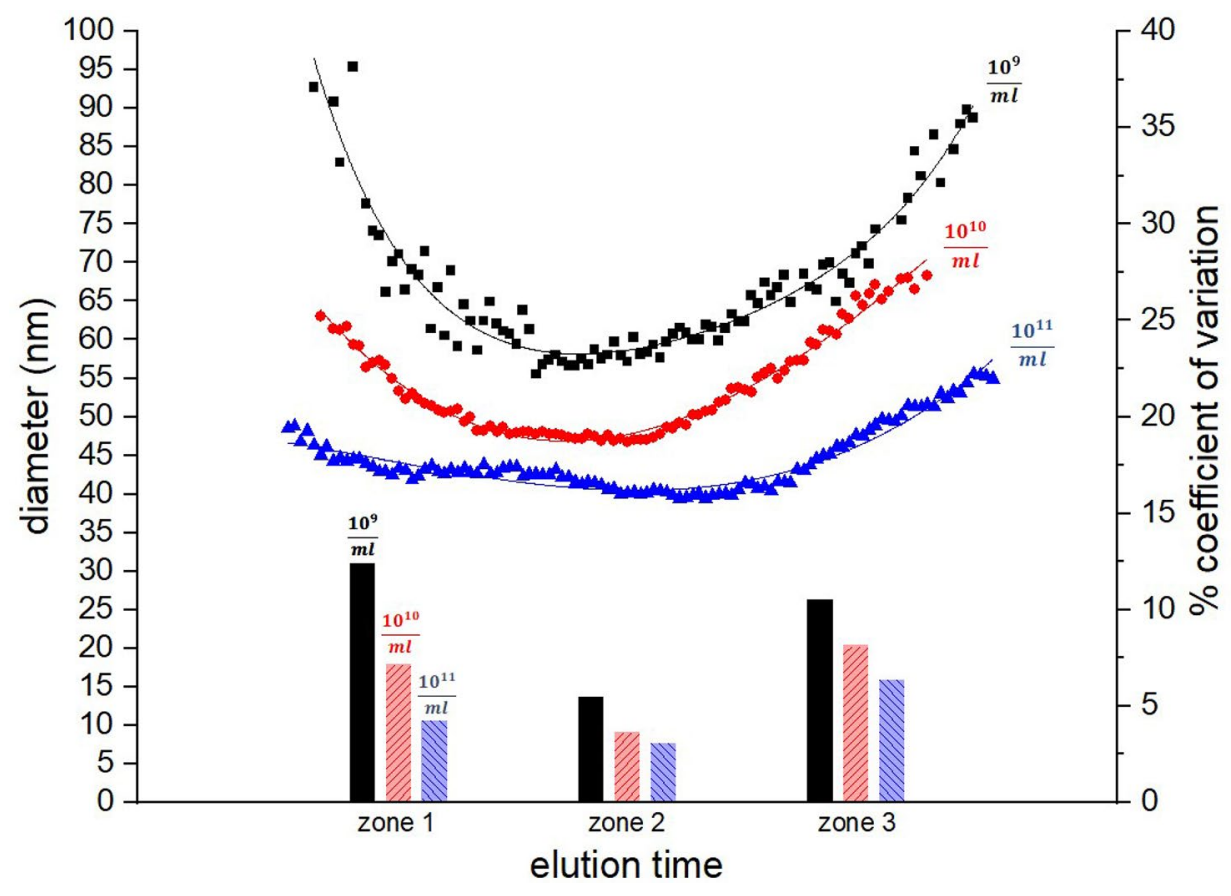

(Fig S1-S6). At the higher initial concentration of $10^{11}$ particles $\mathrm{mL}^{-1}$, the AF4-DLS-UV system provides an accurate sizing of the nanoparticles in suspension returning an average diameter close to the nominal one with low coefficients of variation throughout all the zones identified in the absorbance distribution curves. The apparent size increase at the beginning and at the end of the elution curve starts to appear for an initial working concentration of $10^{10}$ particles $\mathrm{mL}^{-1}$ and becomes progressively more evident as the initial working concentration of the particles in suspension is decreased. At the lowest concentration tested of $10^{9}$ particles $\mathrm{mL}^{-1}$, the DLS returns a noticeable $\mathrm{U}$-shaped distribution for the diameters as a function of the elution time and detects an increase in the size of the particles of at least the $50 \%$ respect with to the nominal size of the particles, even in the region associated with maximum concentration of particles passing through the size detector, i.e., zone 2 . The dependence of the coefficient of variation on the concentration of particles provides a further confirmation of the sizing inaccuracy of the AF4-DLS-UV system, both for suspensions at low initial concentrations and for suspensions at the beginning and end of the elution process. The coefficient of variation quantifies the variability of a set of data by evaluating the level of dispersion of a given set of measurements around the mean. A high coefficient of variation indicates low reliability and stability of the measurements [15]. In our test scenario, the high coefficients of variation exhibited particularly at the beginning and end of the elution process by suspensions with particle concentrations up to $10^{10}$ particles $\mathrm{mL}^{-1}$ provides evidence of the failure of the AF4-DLS-UV system to yield an accurate size characterisation for the population of particles in suspension.

Similar results can be observed analysing the measurements provided by the AF4-DLS-UV system for the suspensions of $60 \mathrm{~nm}$ gold nanoparticles investigated (Fig. 4). Again, the suspension at the lowest initial concentration of $10^{9}$ particles $\mathrm{mL}^{-1}$ exhibits a more obvious U-shaped distribution for the diameters and the highest coefficient of variation in all the regions of absorbance. For suspensions in this concentration range, the average diameter detected by the DLS is at least $35 \%$ higher than the nominal diameter of nanoparticles in suspension.

The concentration-dependence of the sizing ability of the AF4-DLS-UV system demonstrated in our study can be explained by looking at the theoretical principles of the DLS-based sizing analysis. The DLS detector evaluates the diffusion coefficient of particles in suspension quantifying the rate of variation of the light scattered by the suspension over time [16].

Then, the Stokes-Einstein equation is applied to determine the hydrodynamic diameter of the particles:

$D=\frac{K_{b} T}{3 \pi \eta d}$

where $D$ is the diffusion coefficient, $d$ is the diameter of the particle, $K_{b}$ is the Boltzmann constant, $T$ is temperature and $\eta$ is fluid viscosity. Criticism about the accuracy of DLS measurement coupled to AF4 has been already reported in literature for high values of the suspension flow velocity passing through the detector. A fast suspension flow induces 
Fig. 4 Detected diameters of $60 \mathrm{~nm}$ gold nanoparticles (data points and left axis) in suspension at different concentrations as a function of the elution time and coefficient of variation (columns and right axis) for the three absorbance zones identified based on results from AF4-DLS-UV system

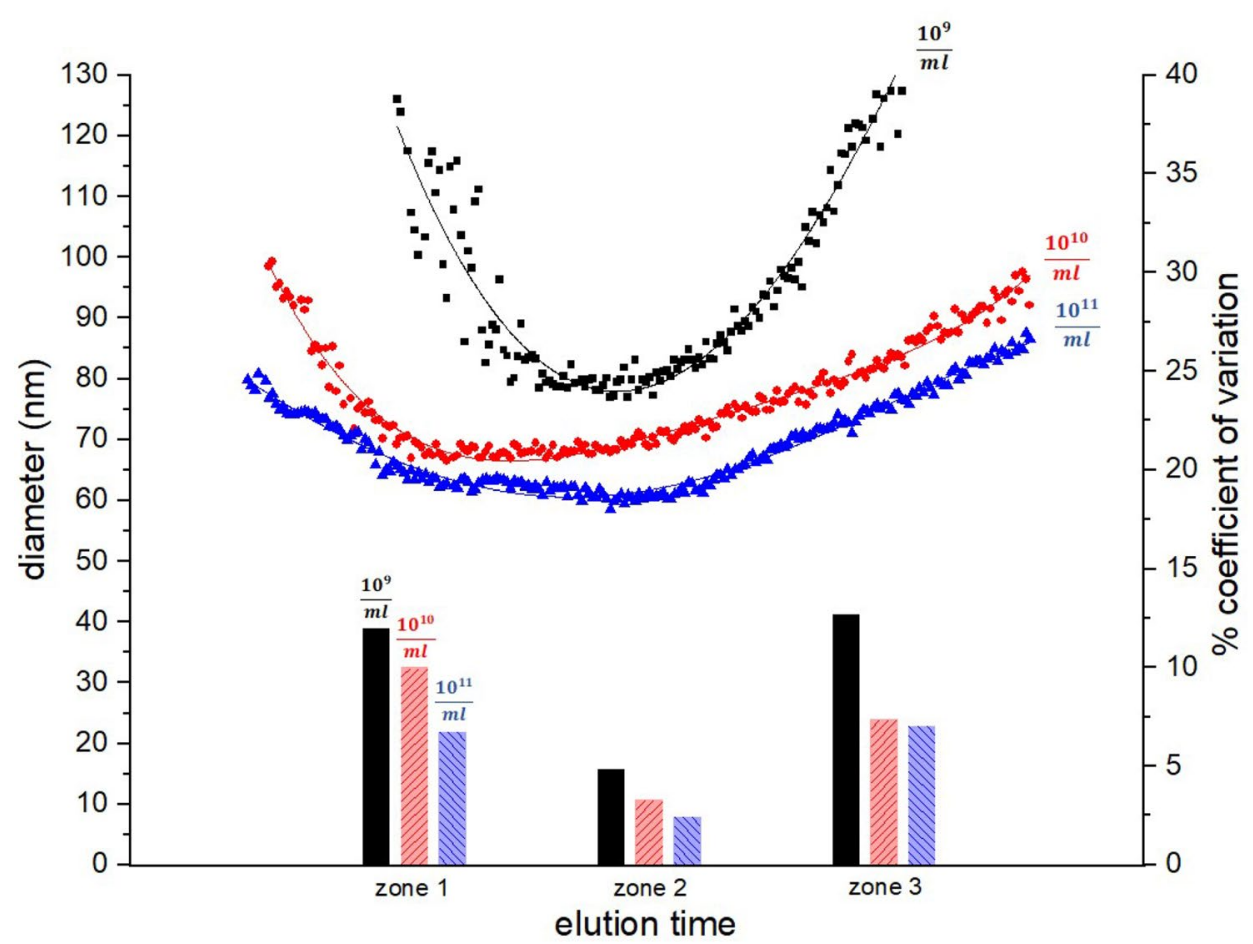

an additional and non-negligible translational component to the natural diffusion process of the particles. The DLS misinterprets this higher diffusion motility as a result of the presence of smaller particles in suspension, returning an inaccurate size distribution of the particles population. However, the effect of the flow rate and particle size used in this study on DLS accuracy has been reported to be negligible [17]. Moreover, our investigation evidences an opposite phenomenon, with the DLS detecting an apparent size increase of the particles in suspension as the concentration is reduced. A number of studies in the literature have demonstrated that the diffusion process of particles at low concentrations is slower than predicted by the Stokes-Einstein equation. Holmberg et al. found using a dynamic light scattering technique that the diffusion coefficient of titanium dioxide nanoparticles is dependent on the mass concentration of particles in solution and is lower than the diffusion coefficient calculated with Stokes-Einstein equation [18]. Similar results were obtained by Tuoriniemi et al. [8] characterising the diffusion behaviour of silica nanoparticles. Coglitore et al. found by tracking single monodispersed gold and polystyrene nanoparticles that, below a critical size and concentration, the diffusion coefficient of the nanoparticles was at least one order of magnitude smaller than the theoretical value predicted by Stokes-Einstein equation and was independent of nanoparticle size and density. The transition from the Stokes-Einstein diffusion regime to the slower diffusion regime has been reported to appear for concentration of particles in the range of $10^{8}-10^{9}$ particles $\mathrm{m}^{-1}[19,20]$.
This evidence demonstrates that, at low concentrations, the nanoparticles do not follow the Stokes-Einstein diffusive regime and the real diffusion coefficient is lower than the theoretical prediction. As a result, the DLS fails to determine the real size of nanoparticles in suspension returning an apparent size increase. In our test scenario, this concentration-dependant diffusion process causes the sizing failure of the AF4-DLS-UV system for suspensions with low concentrations of particles. In fact, at the beginning and end of the elution process, the concentration of particles in suspension is progressively lower as well as their diffusion behaviour. The DLS misinterprets the progressive decrease of the diffusion coefficient as a result of a progressive increase in the size of nanoparticles in suspension, returning a U-shaped curve for the diameter as a function of the concentration of particles. This hypothesis has been confirmed by the CLS analysis performed on the same suspension of nanoparticles. In a CLS analysis, the diffusion behaviour of nanoparticles is not taken into account and the Stokes diameter of particles in suspension is derived from the Stokes sedimentation law through the evaluation of the sedimentation time of particles subjected to a centrifugal force field driving their transport towards the medium [16]. Figure 5 shows that this analysis provided more consistent results between suspensions at different concentration of particles, demonstrating the negligible influence of the concentration of particles on the sizing ability of the CLS both for $40 \mathrm{~nm}$ and $60 \mathrm{~nm}$ suspensions, proving the theoretical limitations of a DLS-based analysis. 
Fig. 5 CLS particle size distributions for the a $40 \mathrm{~nm}$ and $\mathbf{b} 60 \mathrm{~nm}$ suspensions of gold nanoparticles at different concentrations. The absorbance values have been normalised dividing the original values by the relative scale factors shown inset
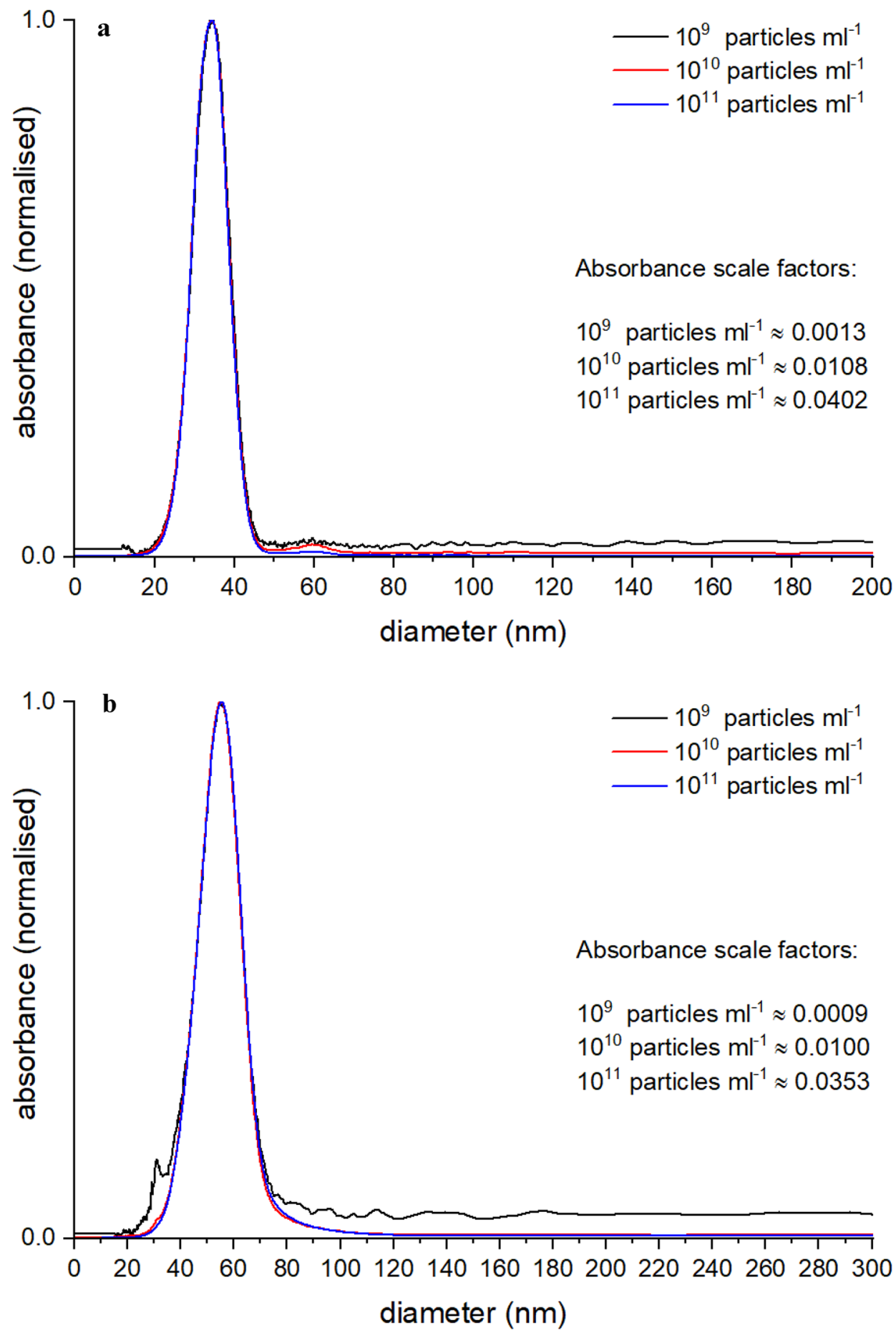

\section{Conclusion}

In this study we have investigated the reliability of the size measurements provided by an AF4 machine coupled with online DLS and UV detectors for suspensions of gold nanoparticles of a known nominal size of $40 \mathrm{~nm}$ and $60 \mathrm{~nm}$ at a range of different concentrations. The results obtained demonstrated that the theoretical limitations and resolution constraints of the DLS analysis compromises the reliability of the size characterisation provided by the AF4-DLS-UV system for suspensions at low concentrations of particles. The deviation of the real diffusion behaviour of nanoparticles at low concentration from the theoretical predictions causes the DLS to detect an apparent and progressive size increase, reducing the accuracy of the size measurements. The concentration-dependant sizing ability of the 
AF4-DLS-UV system demonstrated in this work should be taken into account particularly in biomedical and toxicological investigations, where the correct characterisation of the physical properties of particles in solution is a key prerequisite to assess their potential positive as well as negative impact.

Author contributions FG conducted the experiments under the direct supervision of EAP and JMC. MW, DG and RLS contributed to the design of the study and interpretation of the results. FG prepared the first draft of the manuscript and all authors contributed to the production of the final version. All authors have given approval to the final version of the manuscript.

Funding FG gratefully acknowledges funding from the UK Engineering and Physical Sciences Research Council in the form of a PhD studentship and funding from the European Commission Joint Research Centre (JRC) in the form of a traineeship.

Availability of Data and Material Data sets available upon request.

\section{Compliance with Ethical Standards}

Conflict of interest The authors declare that they have no competing interests.

Open Access This article is licensed under a Creative Commons Attribution 4.0 International License, which permits use, sharing, adaptation, distribution and reproduction in any medium or format, as long as you give appropriate credit to the original author(s) and the source, provide a link to the Creative Commons licence, and indicate if changes were made. The images or other third party material in this article are included in the article's Creative Commons licence, unless indicated otherwise in a credit line to the material. If material is not included in the article's Creative Commons licence and your intended use is not permitted by statutory regulation or exceeds the permitted use, you will need to obtain permission directly from the copyright holder. To view a copy of this licence, visit http://creativecommons.org/licenses/by/4.0/.

\section{References}

1. Messaud FA, Sanderson RD, Runyon JR, Otte T, Pasch H, Williams SKR (2009) An overview on field-flow fractionation techniques and their applications in the separation and characterization of polymers. Prog Polym Sci 34(4):351-368

2. Eskelin K, Lampi M, Meier F, Moldenhauer E, Bamford DH, Oksanen HM (2016) Asymmetric flow field flow fractionation methods for virus purification. J Chromatogr A 1469:108-119

3. Gigault J, Mignard E, Hadri HE, Grassl B (2017) Measurement bias on nanoparticle size characterization by asymmetric flow field-flow fractionation using dynamic light-scattering detection. Chromatographia 80(2):287-294

4. Cho TJ, Hackley VA (2010) Fractionation and characterization of gold nanoparticles in aqueous solution: asymmetric-flow field flow fractionation with MALS, DLS, and UV-Vis detection. Anal Bioanal Chem 398(5):2003-2018

5. Caputo F, Arnould A, Bacia M, Ling WL, Rustique E, Texier I, Mello AP, Couffin AC (2019) Measuring particle size distribution by asymmetric flow field flow fractionation: a powerful method for the preclinical characterization of lipid-based nanoparticles. Mol Pharm 16(2):756-767

6. Wolfgang H, Nguyen TKT, Jenny A, David GF (2007) Determination of size and concentration of gold nanoparticles from UV-Vis spectra. Anal Chem 79(11):4215-4221

7. Jang M, Lee S, Hwang YS (2015) Characterization of silver nanoparticles under environmentally relevant conditions using asymmetrical flow field-flow fractionation (AF4). PLoS ONE 10(11):e0143149

8. Tuoriniemi J, Johnsson AJH, Holmberg JP, Gustafsson S, GallegoUrrea JA, Olsson E, Pettersson JBC, Hassellöv M (2014) Intermethod comparison of the particle size distributions of colloidal silica nanoparticles. Sci Technol Adv Mater 15(3):035009

9. António DC, Cascio C, Gilliland D, Nogueira AJ, Rossi F, Calzolai L (2016) Characterization of silver nanoparticles-alginate complexes by combined size separation and size measurement techniques. Biointerphases 11(4):04B309

10. Palais C, Chichester JA, Manceva S, Yusibov V, Arvinte T (2015) Influence of protein formulation and carrier solution on asymmetrical flow field-flow fractionation: a case study of the plantproduced recombinant anthrax protective antigen pp-PA83. J Pharm Sci 104(2):612-617

11. Bednar AJ, Poda AR, Mitrano DM, Kennedy AJ, Gray EP, Ranville JF, Hayes CA, Crocker FH, Steevens JA (2013) Comparison of on-line detectors for field flow fractionation analysis of nanomaterials. Talanta 104:140-148

12. Humbert C, Pluchery O, Lacaze E, Tadjeddine A, Busson B (2013) Optical spectroscopy of functionalized gold nanoparticles assemblies as a function of the surface coverage. Gold Bull 46(4):299-309

13. Cascio C, Geiss O, Franchini F, Ojea-Jimenez I, Rossi F, Gilliland D et al (2015) Detection, quantification and derivation of number size distribution of silver nanoparticles in antimicrobial consumer products. J Anal At Spectrom 30(6):1255-1265

14. Geiss O, Cascio C, Gilliland D, Franchini F, Barrero-Moreno J (2013) Size and mass determination of silver nanoparticles in an aqueous matrix using asymmetric flow field flow fractionation coupled to inductively coupled plasma mass spectrometer and ultraviolet-visible detectors. J Chromatogr A 1321:100-108

15. Shechtman O (2013) The coefficient of variation as an index of measurement reliability. In: Doi S, Williams G (eds) Methods of clinical epidemiology. Springer series on epidemiology and public health. Berlin, Springer, pp 39-49

16. Braun A, Couteau O, Franks K, Kestens V, Roebben G, Lamberty A, Linsinger TPJ (2011) Validation of dynamic light scattering and centrifugal liquid sedimentation methods for nanoparticle characterisation. Adv Powder Technol 22(6):766-770

17. Sitar S, Vezocňik V, Macěk P, Kogej K, Pahovnik D, Žagar E (2017) Pitfalls in size characterization of soft particles by dynamic light scattering online coupled to asymmetrical flow field-flow fractionation. Anal Chem 89(21):11744-11752

18. Perez Holmberg J, Abbas Z, Ahlberg E, Hassellöv M, Bergenholtz J (2011) Nonlinear concentration dependence of the collective diffusion coefficient of $\mathrm{TiO} 2$ nanoparticle dispersions. J Phys Chem C 115(28):13609-13616

19. Coglitore D, Edwardson SP, Macko P, Patterson EA, Whelan M (2017) Transition from fractional to classical Stokes-Einstein behaviour in simple fluids. R Soc Open Sci 4(12): 170507

20. Giorgi F, Coglitore D, Curran JM, Gilliland D, Macko P, Whelan M, Worth A, Patterson EA (2019) The influence of 
inter-particle forces on diffusion at the nanoscale. Sci Rep. https ://doi.org/10.1038/s41598-019-48754-5

21. Nanolitics. Field-Flow Fractionation. https://www.nanolytics.de/ en/other_methods_colloidal_analytics/field-flow_fractionation. Accessed 15 Apr 2020.
Publisher's Note Springer Nature remains neutral with regard to jurisdictional claims in published maps and institutional affiliations. 